\title{
Holoprosencephaly-radial heart renal anomalies syndrome
}

INSERM

\section{Source}

INSERM. (1999). Orphanet: an online rare disease and orphan drug data base.

Holoprosencephaly-radial heart renal anomalies syndrome. ORPHA:3186

Holoprosencephaly-radial heart renal anomalies syndrome is characterised by

holoprosencephaly, predominantly radial limb deficiency (absent thumbs, phocomelia),

heart defects, kidney malformations and absence of gallbladder. 